halten auch bei $23^{\circ}$ feststellten. $\mathrm{Zu}$ diesen Befunden' passen gut die Beobachtungen von JoEL (17), der während der Lipolyse bei $37^{\circ}$ einen deutlich höheren Sauerstoffverbrauch feststellte als bei $8^{\circ}$. Für das experimentelle Vorgehen bei der in-vitro-Lipolyse läßt sich aus diesen Befunden ableiten, daß bei der hormoninduzierten Lipolyse eine genaue Temperatureinstellung $z$ wischen $30^{\circ}$ und $40^{\circ}$ nicht erforderlich ist. Die Feststellung von MosINGER (9), daß bei nicht maximaler Adrenalinkonzentration im Medium die Freisetzung freier Fettsäuren linear zur Zeit verläuft, trifft nach unseren Untersuchungen auch für die Spontanlipolyse und die durch ACTH stimulierte Lipolyse zu. Da jedoch im allgemeinen die Inkubation nicht länger als $3 \mathrm{Stdn}$. durchgeführt wird, gilt das auch für maximale Hormon- dosen; nur ist hierbei der wesentlich steilere Anstieg der freien Fettsäuren bereits in der ersten Std. zu berücksichtigen.

Wenn auch alle Untersuchungen am epididymalen Fettgewebe der Ratte vorgenommen wurden, ist nach den Mitteilungen aus der Literatur und unseren eigenen Ergebnissen eine Übertragung dieser Lipolysebedingungen auf andere Fettgewebsarten und andere Species möglich.

Möglicherweise läßt sich die geringere Ansprechbarkeit des menschlichen Fettgewebes auf lipolytische Hormone $(9,18)$ durch Modifikation einzelner Inkubationsbedingungen verbessern.

Herrn Dr. MrLesch, Farbwerke Hoechst, danken wir für die Überlassung von Rinderalbumin Behring.

\title{
Literatur
}

1. Gordon, R. S. und A. Cherkers, Proc. Soc. exp. Biol. Med. 97, 150 (1958). - 2. Whrte, J. E. und F. L. ENGEL, Proc. Soc. exp. Biol. Med. 99, 375 (1958). - 3. Schwandt, P., Th. Hartmann und H. J. Kari, Zschr. exper. Med. 143, 79 (1967). - 4. CAMPBell, J., A. D. Marticcr und G. R. Green, Biochem. J. 93, 183 (1964). 5. Dole, V. P. und H. Meinertz, J. biol. Chemistry 235, 2595 (1960). - 6. Rudman, D., L. A. Garcia, S. J. Brown, M. F. Malkin und W. Perl, J. Lipid Res. 5, 28 (1964). - 7. LynN, S., R. M. MACLeAd und R. H. Brown, J. biol. Chemistry 235, 1904 (1960). - 8. Hirsch, J., in: Handbook of Physiology, Section 5, 455 (1965), Williams \& Wilkins Company, Baltimore, Maryland, USA. - 9. Mosinger, B., in: Handbook of Physiology, Section 5 ,
601 (1965). - 10. Christophe, J.: Contribution à la biochemie des obésités experimentales, Editions Arscia S. A., Bruxelles, 1961. 11. Lopez, E., J. E. White und F. L. ENGeL, J. biol. Chemistry 234, 2254 (1959). - 12. Rodbel, M., in: Handbook of Physiology, Section 5, 471 (1965). - 13. Sutherland, E. W. und G. A. RoBison, Pharmacol. Rev. 18, 145 (1966). - 14. WINEGRAD, A. J., Vitamins and Horm., N. Y. 20, 141 (1962). - 15. Rudman, D., J. Lipid Res. 4, 119 (1963). - 16. VaughaN, M., in: Handbook of Physiology, Section 5, 239 (1965). - 17. JoEL, C. D., in: Handbook of Physiology, Section 5, 59 (1965). - 18. Schwandt, P. und Th. HartmanN, unveröffentlichte Versuche.

\section{Vorkommen von UDP-Glucuronyltransferasen in der Magenschleimhaut des Menschen}

\author{
Von W. HofFManN und H. Breuer \\ Aus der Abteilung für Klinische Chemie und Biochemie der Cbirurgischen Universitätsklinik und Poliklinik Bonn \\ (Direktor: Prof. Dr. A. Gütgemann)
}

(Eingegangen am 24. August 1967)

Nach Inkubation von $\left[4^{14} \mathrm{C}\right] 17 \beta$-Östradiol mit der Cytoplasma-Fraktion (105000 $\mathrm{g}$-Uberstand) der pylorusnahen Magenschleimhaut des Menschen in Gegenwart von Uridin-5'-diphospho-glucuronat konnte $\left[4-{ }^{14} \mathrm{C}\right] 17 \beta$-Östradiol-17 $\beta$-monoglucuronid nachgewiesen werden; $\left[4^{14} \mathrm{C}\right] \mathrm{O}$ striol ergab unter den gleichen Bedingungen $\left[4{ }^{14} \mathrm{C}\right]$ Östriol-17 $\beta$-monoglucuronid. In Versuchen mit der MikrosomenFraktion der Magenschleimhaut entstanden ebenfalls $\left[{ }^{14} \mathrm{C}\right] 17 \beta$-Östradiol-17 $\beta$-monoglucuronid und $\left[4-{ }^{14} \mathrm{C}\right]$ Östriol-17 $\beta$-monoglucuronid; außerdem wurde $\left[4{ }^{14} \mathrm{C}\right]$ Östriol-16 $\alpha$-monoglücuronid gebildet. $\left[4{ }^{14} \mathrm{C}\right]$ Ostron wurde weder durch die Cytoplasma- noch durch die Mikrosomen-Fraktion glucuronidiert. Die Versuche zeigen, daß in der Magenschleimhaut des Menschen cytoplasmatische (lösliche) und mikrosomale UDP-Glucuronyltransferasen vorhanden sind. Die Glucuronyltransferasen besitzen eine ausgeprägte Gruppenspezifität und greifen nur alkoholische Hydroxylgruppen phenolischer Steroide an. Demnach bestehen zwischen den Glucuronyltransferasen der Magenschleimhaut und der Dünndarmschleimhaut des Menschen Unterschiede.

The enzymic formation of $\left[4{ }^{14} \mathrm{C}\right] 17 \beta$-oestradiol $17 \beta$-monoglucuronide was demonstrated after incubation of $\left[4{ }^{14} \mathrm{C}\right] 17 \beta$-oestradiol with the cytoplasmic fraction ( $105000 \mathrm{~g}$ supernatant) of human gastric mucosa in the presence of uridine diphosphate glucuronic acid. Under similar conditions, $\left[4^{14} \mathrm{C}\right]$ oestriol yielded $\left[4^{14} \mathrm{C}\right]$ oestriol $17 \beta$-monoglucuronide. Similarly, in experiments with the microsomal fraction, $[4-14 \mathrm{C}] 17 \beta$-oestradiol $17 \beta$-monoglucuronide and $\left[4{ }^{14} \mathrm{C}\right]$ oestriol $17 \beta$-monoglucuronide were formed from $\left[4{ }^{14} \mathrm{C}\right] 17 \beta$-oestradiol and $\left[4{ }^{14} \mathrm{C}\right]$ oestriol, respectively; in addition, $\left[{ }^{14} \mathrm{C}\right]$ oestriol $16 \alpha$-monoglucuronide was detected. No glucuronidation of $[4-14 \mathrm{C}]$ oestrone occurred in either the cytoplasmic or the microsomal fractions. These experiments demonstrate that the human gastric mucosa contains soluble as well as microsomal UDP-glucuronyltransferases. The glucuronyltransferases possess a pronounced substrate specifity and attack only alcoholic hydroxyl groups of phenolic steroids. The results reported here show that differences exist between the glucuronyltransferases of the gastric mucosa and the intestinal mucosa of man. 
In früheren Arbeiten konnte gezeigt werden, daß die Bildung von $C_{19^{-}}$und $C_{18^{-}}$-Steroidglucuroniden durch spezifische UDP-Glucuronat-Glucuronyltransferasen (EC 2.4.1.17) katalysiert wird (1-6). Während bis vor kurzem angenommen wurde, daß die Glucuronyltransferasen ausschließlich in den Mikrosomen-Fraktionen der verschiedenen Organe lokalisiert sind (vgl. (7)), gelang inzwischen der Nachweis löslicher Glucuronyltransferasen im Groundplasma (150000 $\mathrm{g}$-Überstand) des Dünndarms vom Menschen (4-6). Im Hinblick auf die Bedeutung, die dem gastrointestinalen Abschnitt des Verdauungstraktes bei der Bildung von Glucuroniden zugesprochen wird $(7,8)$, schien es von Interesse, Untersuchungen über das Vorkommen, die Substratspezifität und die intracelluläre Verteilung von Glucuronyltransferasen im Magen des Menschen durchzuführen. Wie im folgenden gezeigt wird, kommen in der Magenschleimhaut des Menschen sowohl lösliche als auch mikrosomale Steroid-Glucuronyltransferasen vor, die - im Gegensatz zu den entsprechenden Enzymen der Dünndarmmucosa - eine Gruppenspezifität aufweisen.

\section{Methodik}

Steroide

[4 $\left.{ }^{14} \mathrm{C}\right]$ Östron [Östra-1.3.5(10)-trien-3-ol-17-on] (spez. Aktivität $29,5 \mathrm{mC} / \mathrm{mMol}$ )

$\left[4^{14} \mathrm{C}\right] 17 \beta$-Östradiol [Östra-1.3.5(10)-trien-3.17 $\beta$-diol] (spez. Aktivität $31,8 \mathrm{mC} / \mathrm{mMol}$ )

[4-14 C]Östriol [Östra-1.3.5(10)-trien-3.16 $\alpha .17 \beta$-triol] (spez. Aktivität $29,4 \mathrm{mC} / \mathrm{mMol}$ ) und

[6.7-3 $\left.{ }^{3}\right]$ Östriol (spez. Aktivität $1610 \mathrm{mC} / \mathrm{mMol}$ )

wurden vom Radiochemical Centre, Amersham, England, bezogen. Alle radioaktiven Steroide wurden vor den Versuchen papierchromatographisch auf Reinheit geprüft.

Östriol-17 $\beta$-monoglucuronid [Östra-1.3.5(10)-trien-3.16 $\alpha .17 \beta$-triol1 i $\beta$-yl- $\beta$-D-glucopyranuronat]

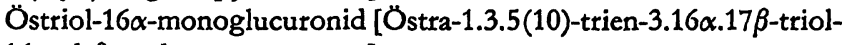
$16 \alpha$-yl- $\beta$-D-glucopyranuronat],

Östriol-3-monoglucuronid [Östra-1.3.5(10)-trien-3.16 $\alpha .17 \beta$-triol-3yl- $\beta$-D-glucopyranuronat] und

Östron-3-monoglucuronid [Östra-1.3.5(10)-trien-3-ol-17-on-3-y]$\beta$-D-glucopyranuronat]

wurden von Dr. A. E. Kellie, London, zur Verfügung gestellt. $17 \beta$-Östradiol-17 $\beta$-monoglucuronid [Östra-1.3.5(10)-trien-3.17 $\beta$ diol-17 $\beta$-yl- $\beta$-D-glucopyranuronat]

stammte von Prof. H. H. Woriz, Boston.

\section{Reagentien und Lösungsmittel}

Mit Ausnahme von Folin-Crocalteus-Reagenz waren alle Reagenzien von p. a. Reinheitsgrad (E. Merck, Darmstadt). Die organischen Lösungsmittel wurden vor Gebrauch destilliert. Uridin-5'-diphospho-glucuronat (80\% di-Natriumsalz) wurde von C. F. Boehringer \& Soehne, Mannheim, bezogen.

\section{Gewebe}

Alle Versuche wurden mit Magenschleimhaut vom Menschen durchgeführt, die bei der Resektion distaler Magenabschnitte wegen eines Ulcus gewonnen wurde. Das Gewebe stammte aus dem pylorusnahen Magen-Antrum; die histologische Untersuchung zeigte eine regelrechte Beschaffenheit der Magenschleimhaut. Die Zeit zwischen der Gewebsentnahme und dem Beginn der Aufarbeitung betrug 5-10 Min.

\section{Zellfraktionierung}

Die Magenschleimhaut wurde in Höhe der Submucosa abpräpariert, in einem Fleischwolf zerkleinert und in 0,25M Rohrzucker-Lösung in einem Glashomogenisator bei $0^{\circ}$ homogenisiert. Das 50 proz. Homogenat wurde zunächst $20 \mathrm{Min}$. bei $800 \mathrm{~g}$ in einer SpincoZentrifuge L-2 zentrifugiert. Anschließend wurde der Überstand $45 \mathrm{Min}$. bei $20000 \mathrm{~g}$ zentrifugiert. Der $20000 \mathrm{~g}$-Uberstand wurde $60 \mathrm{Min}$. bei $105000 \mathrm{~g}$ zentrifugiert. Der $105000 \mathrm{~g}$-Úberstand wurde vom Sediment getrennt und zweimal $30 \mathrm{Min}$. bei $105000 \mathrm{~g}$ rezentrifugicrt. $2 \mathrm{ml}$ des so gewonnenen $105000 \mathrm{~g}$-Uberstandes enthielten die Cytoplasma-Fraktion aus $1 \mathrm{~g}$ Frischgewebe. Das Sediment der 1. Zentrifugation bei $105000 \mathrm{~g}$ wurde in $0,25 \mathrm{M}$ Rohtzucker-Lösung resuspendiert und erneut 30 Min. bei $105000 \mathrm{~g}$ zentrifugiert; dieser Schritt wurde einmal wiederholt. Die so gewonnerie Mikrosomen-Fraktion wurde in $0,15 \mathrm{M}$ Soerensen-Phosphat-Puffer, pH 6,8, aufgenommen, so daß $2 \mathrm{ml}$ die MikrosomenFraktion aus $1 \mathrm{~g}$ Frischgewebe enthielten.

\section{Inkeubationsbedingungen}

Alle Versuche wurden bei $37^{\circ}$ für $120 \mathrm{Min}$. in einem Schüttelthermostaten unter Luft durchgeführt. Jeweils $1 \mu \mathrm{C}\left[\right.$ [4-14 $\left.^{14}\right]$ Steroid, gelöst in $0,01 \mathrm{ml}$ Propylenglykol, wurde in Gegenwart von $4 \mathrm{mg}$ Uridin-5'-diphospho-glucuronat mit $2 \mathrm{ml}$ der MikrosomenFraktion bzw. $2 \mathrm{ml}$ der Cytoplasma-Fraktion und $4 \mathrm{ml}$ eines 0,15 Soerensen-Phosphat-Puffers, $\mathrm{pH}$ 6,8, inkubiert. Das Gesamtvolumen der Inkubationsansätze betrug $6 \mathrm{ml}$.

\section{Aufarbeitung der Versucbsansätze}

Die Lösungen wurden dreimal mit jeweils $5 \mathrm{ml}$ Äther/Chloroform $(3: 1 \mathrm{v} / \mathrm{v})$ extrahiert, die Extrakte vereinigt und bei $35^{\circ}$ eingedampft (freie Steroide). Zur Gewinnung der Glucuronide wurden die wäßr. Phasen jeweils mit $14 \mathrm{~m} / 96 \mathrm{proz}$. Äthanol versetzt und über Nacht stehengelassen. Nach Zentrifugation wurden die Lösungen von den Niederschlägen dekantiert und die Niederschläge zweimal mit je $2 \mathrm{ml} 70$ proz. Äthanol gewaschen. Die wäßr.-äthanolischen Lösungen wurden vereinigt und zur weitgehenden Entfernung von Äthanol bei $50^{\circ}$ auf etwas weniger als die Hälfte eingeengt. Die wäßr. Phasen wurden zwiveimal mit je $10 \mathrm{ml} n$-Butanol extrahiert; sofern Emulsionen entstanden, wurde zentrifugiert. Die butanolischen Lösungen wurden mit einem Rotationsverdampfer bei $50^{\circ}$ zur Trockne eingedampft (Steroidglucuronide) und die Extraktrückstände in Methanol/Wasser $(1: 1 \mathrm{v} / \mathrm{v})$ aufgenommen.

\section{Papierchromatographie}

Die Papierchromatographie der freien Steroide erfolgte bei $25-27^{\circ}$ auf formamidimprägniertem Papier (Schleicher \& Schüll 2043b $\mathrm{Mgl}$ ) in den Systemen Monochlorbenzol und Chloroform/Äthylacetat $(5: 1 \mathrm{v} / \mathrm{v})$. Die Steroidglucuronide wurden bei $35^{\circ}$ im System Essigsäure/Wasser/tert. Butanol/Dichloräthan (6:14: $5: 15$ $\nabla / v)$ chromatographiert, nachdem die Papierchromatogramme über Nacht äquilibriert worden waren. Die Lokalisierung der freien Steroide und der Steroidglucuronide erfolgte durch Messung der Radioaktivität und Vergleich der Wanderungsgeschwindigkeiten mit denjenigen der authentischen Referenzsubstanzen. Sowohl die freien Östrogene als auch die phenolischen Steroidglucuronide wurden mit Folin-Crocalteus-Reagenz (9) sichtbar gemacht. Zur weiteren Identifizierung wurden die Steroidglucuronide von den Papierchromatogrammen eluiert.

\section{Hydrolyse der Steroidglucuronide}

Die phenolischen Steroidglucuronide wurden in $5 \mathrm{ml}$ eines $0,15 \mathrm{M}$ Acetat-Puffers, $\mathrm{pH} 5,2$, mit $0 ; 5 \mathrm{ml}$ eines Extraktes aus Helix pomatia ${ }^{1}$ ) (50000 Fishman-Einheiten $\beta$-Glucuronidase) $24 \mathrm{Stdn}$. bei $37^{\circ}$ inkubiert. Die freigesetzten Steroide wurden dreimal mit je $10 \mathrm{~m} l$ Äther/Chloroform $(3: 1 \mathrm{v} / \mathrm{v})$ extrahiert, die Extrakte vereinigt und bei $35^{\circ}$ eingedampft. Die Rückstände wüurden in Methanol aufgenommen und der Papierchromatographie unterworfen.

\section{Quantitative Bestimmungen}

Die aus den Inkubationsansätzen extrahierten freien Steroide wurden in $5 \mathrm{ml}$ einer Scintillations-Lösung (enthaltend $5 \mathrm{~g} / \mathrm{l} 2,5$ Diphenyloxazol [PPO] und 0,3 g/l 1,4-bis-2-(4-Methyl-5-phenyloxazolyl)-benzol [Dimethyl-POPOP]) mit Hilfe eines Tricarb-

1) Hersteller: L'Industrie biologique Française S. A., Gennevilliers, Seine. 
Scintillations-Spektrometers Modell 3003 der Fa. Packard mit extcrnem Standard quantitativ bestimmt; die Zählausbeute für ${ }^{14} \mathrm{C}$ beträgt $81 \%$. Die quantitative Bestimmung der Steroidglucuronide erfolgte nach Papierchromatographie durch Auszählung der Radioaktivität auf $5 \mathrm{~cm}$ breiten und $50 \mathrm{~cm}$ langen Papierstreifen mit einem Radiopapierchromatographen Modell 7201 der Fa. Packard, ausgerüstet mit einem fensterlosen Helium-Butan-Durch-

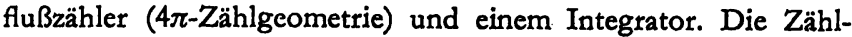
ausbeute für ${ }^{14} \mathrm{C}$ beträgt $27 \%$. Die Stickstoff bestimmungen erfolgten nach KJELDAHL.

\section{Ergebnisse}

\section{Identifizierung der Steroidglucuronide}

Nach Inkubation von $1 \mu \mathrm{C}[4-14 \mathrm{C}] 17 \beta$-Östradiol mit der Mikrosomen-Fraktion der Magenmucosa in Gegenwart von Uridin-5'-diphospho-glucuronat wurde ein wasserlöslicher Metabolit nachgewiesen (A-1), der bei der Papierchromatographie im System Essigsäure/ Wasser/tert. Butanol/Dichloräthan den gleichen $R_{\mathrm{F}}$ Wert $(0,71)$ wie $17 \beta$-Östradiol-17 $\beta$-monoglucuronid zeigte. Dieser Wert liegt etwas höher als der in einer früheren Arbeit (10) angegebene $R_{\mathrm{F}}$-Wert für $17 \beta$ Östradiol-17 $\beta$-monoglucuronid $(0,65)$. Der Unterschied $z$ wischen den beiden $R_{F}$-Werten ist durch die verschiedenen Arbeitstemperaturen $\left(20-25^{\circ}\right.$ bzw. $\left.35^{\circ}\right)$ bedingt. Nach Elution vom Papier wurde A-1 enzymatisch hydrolysiert und das freigesetzte Aglykon auf formamidimprägniertem Papier mit Monochlorbenzol chromatographiert; die radioaktive Verbindung hatte den gleichen $R_{\mathrm{F}}$-Wert $(0,39)$ wie authentisches $17 \beta$ Östradiol. Demnach handelt es sich bei A-1 um $17 \beta-$ Östradiol-17 $\beta$-monoglucuronid.

Nach Inkubation von $1 \mu \mathrm{C}\left[4^{14} \mathrm{C}\right]$ Östriol mit der Mikrosomen-Fraktion entstanden unter den gleichen Bedingungen wie oben angegeben zwei wasserlösliche Metaboliten (B-1 und B-2), die sich papierchromatographisch im System Essigsäure/Wasser/tert. Butanol/Dichloräthan gut voneinander trennen ließen und deren $R_{\mathrm{F}}$-Werte mit denjenigen von Östriol-16 $\alpha$-monoglucuronid $(0,51$; B-1) und von Östriol-17 $\beta$-monoglucuronid $(0,59 ; \mathrm{B}-2)$ übereinstimmten. Nach getrennter Elution vom Papier wurden B-1 und B-2 der enzymatischen Hydrolyse unterworfen; die dabei freigesetzten radioaktiven Agly- kone zeigten nach Chromatographie auf formamidimprägniertem Papier im System Chloroform/Äthylacetat $(5: 1 \mathrm{v} / \mathrm{v})$ die gleichen Wanderungsgeschwindigkeiten $(25,6 \mathrm{~cm} / 15 \mathrm{Stdn}$.) wie authentisches Östriol. Daraus geht hervor, daß B-1 mit Östriol-16 $\alpha$-monoglucuronid und B-2 mit Östriol-17 $\beta$-monoglucuronid identisch ist. Nach Inkubation von $1 \mu \mathrm{C}\left[4^{-14} \mathrm{C}\right]$ Östron mit der Mikrosomen-Fraktion konnten keine wasserlöslichen Metaboliten nachgewiesen werden; die gesamte eingesetzte Radioaktivität befand sich in der freien Steroid-Fraktion. Demnach hatte keine Glucuronidierung der phenolischen Hydroxylgruppe von Östron stattgefunden.

In den Inkubationsversuchen mit der Cytoplasma-Fraktion ergaben sich folgende Befunde: $17 \beta$-Östradiol wurde zu 17 $\beta$-Östradiol-17 $\beta$-monoglucuronid konjugiert, während aus Östriol nur Östriol-17 $\beta$-monoglucuronid, nicht aber Östriol-16 $\alpha$-monoglucuronid entstand. Die Identifizierung von $17 \beta$-Östradiol-17 $\beta$-monoglucuronid und Östriol-17 $\beta$-monoglucuronid erfolgte wie oben beschrieben. Östron wurde - ähnlich wie in den Versuchen mit der Mikrosomen-Fraktion - durch die Cytoplasma-Fraktion nicht glucuronidiert.

\section{Quantitative Untersucbungen}

Die Ergebnisse der quantitativen Untersuchungen sind in der Tabelle 1 zusammengefaßt. Bezogen auf die eingesetzte Eiweißmenge betrug die Bildungsrate für $17 \beta$ Östradiol-17 $\beta$-monoglucuronid (ausgedrückt in $\mathrm{nMol} / \mathrm{g}$ Eiweiß) 250 bzw. 258 in der Mikrosomen-Fraktion und 11,2 bzw. 8,8 in der Cytoplasma-Fraktion. Weniger deutlich ausgeprägt waren die Unterschiede in der Bildungsrate für Östriol-17 $\beta$-monoglucuronid; sie betrugen in der Mikrosomen-Fraktion 59,0 bzw. 24,5 und in der Cytoplasma-Fraktion 10,5 bzw. 28,8. Die Bildungsraten für Östriol-16 $\alpha$-monoglucuronid in der Mikrosomen Fraktion beliefen sich auf 260 (Versuch G-6) bzw. 71 (Versuch G-7).

\section{Diskussion}

In der vorliegenden Arbeit konnte gezeigt werden, daß die menschliche Magenschleimhaut Glucuronyltrans-

Tab. 1 Bildung von. Östrogenmonoglucuroniden in verschiedenen Zellfraktionen der pylorusnahen Magenschleimhaut des Menschen. Es wurden jeweils
$1 \mu \mathrm{C}$ [4-14C]Steroid mit der Zellfraktion aus $1 \mathrm{~g}$ Frischgewicht inkubiert. Inkubationsbedingungen siehe Methodik. Die quantitative Bestimmung

\begin{tabular}{|c|c|c|c|c|c|c|c|}
\hline $\begin{array}{l}\text { Versuch } \\
\text { Nr. }\end{array}$ & Zellfraktion & $\begin{array}{c}\text { Eiweißgehalt } \\
\text { der inkubierten } \\
\text { Zellfraktionen } \\
\text { (mg) }\end{array}$ & Steroid ${ }^{\text {Inkubiert }}$ & $\begin{array}{l}\text { Menge } \\
\text { (nMol) }\end{array}$ & $\begin{array}{c}\text { Nachgewiesen } \\
\text { Steroidglucuronid }\end{array}$ & $\begin{array}{l}\text { Menge } \\
\text { (nMol) }\end{array}$ & $\begin{array}{c}\text { Gesamt- } \\
\text { wieder- } \\
\text { findung }{ }^{1)} \\
\%\end{array}$ \\
\hline G-6 & $\begin{array}{l}105000 \mathrm{~g}= \\
\text { Uberständ }\end{array}$ & $\begin{array}{l}42,0 \\
42,0 \\
42,0\end{array}$ & 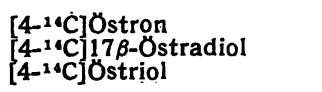 & $\begin{array}{l}33,9 \\
31,4 \\
34,0\end{array}$ & $\begin{array}{l}\text { kein } \\
17 \beta-\text { Monoglucuronid } \\
17 \beta-\text { Monoglucuronid }\end{array}$ & $\begin{array}{l}0 \\
0,47 \\
0,44\end{array}$ & $\begin{array}{l}91 \\
91 \\
93\end{array}$ \\
\hline & $\begin{array}{l}\text { Mikrosomen- } \\
\text { Fraktion }\end{array}$ & $\begin{array}{l}12,0 \\
12,0 \\
12,0\end{array}$ & $\begin{array}{l}{[4-14 C] \text { Stron }} \\
{[4-1 C C] 17 \beta \text {-Ǒstradiol }} \\
{[4-14 C] \text { Ootriol }}\end{array}$ & $\begin{array}{l}33,9 \\
31,4 \\
34,0\end{array}$ & $\begin{array}{l}\text { kein } \\
17 \beta \text {-Monoglucuronid } \\
16 x-\text { Monoglucuronid } \\
17 \beta \text {-Monoglucuronid }\end{array}$ & $\begin{array}{l}0 \\
3,01 \\
3,10 \\
0,71\end{array}$ & $\begin{array}{l}98 \\
96 \\
93 \\
93\end{array}$ \\
\hline \multirow[t]{2}{*}{ G-7 } & $\begin{array}{l}105000 \mathrm{~g}- \\
\text { Ưberstand }\end{array}$ & $\begin{array}{l}32,0 \\
32,0 \\
32,0\end{array}$ & $\begin{array}{l}{[4-14 \mathrm{C}] \text { Ostron }} \\
4-1 * \mathrm{C}] 17 \beta \text {-Östradiol } \\
4-14 \mathrm{C}] \text { Ötriol }\end{array}$ & $\begin{array}{l}33,9 \\
31,4 \\
34,0\end{array}$ & $\begin{array}{l}\text { kein } \\
17 \beta-\text { Monoglucuronid } \\
17 \beta-\text { Monoglucuronid }\end{array}$ & $\begin{array}{l}0 \\
0,28 \\
0,92\end{array}$ & $\begin{array}{l}94 \\
93 \\
95\end{array}$ \\
\hline & $\begin{array}{l}\text { Mikrosomen- } \\
\text { Fraktion }\end{array}$ & $\begin{array}{l}8,2 \\
8,2 \\
8,2\end{array}$ & $\begin{array}{l}{[4-14 \mathrm{C}] \text { Ostron }} \\
{[4-14 \mathrm{C}] 17 \beta-O \text { stradiol }} \\
{[4-14 \mathrm{C}[\mathrm{O} \text { striol }}\end{array}$ & $\begin{array}{l}33,9 \\
31,4 \\
34,0\end{array}$ & $\begin{array}{l}\text { kein } \\
17 \beta \text {-Monoglucuronid } \\
16 \alpha-\text { Monoglucuronid } \\
17 \beta \text {-Monoglucuronid }\end{array}$ & $\begin{array}{l}0 \\
2,12 \\
0,58 \\
0,20\end{array}$ & $\begin{array}{l}92 \\
97 \\
89 \\
89\end{array}$ \\
\hline
\end{tabular}

1) Einschließlich der freien Steroide. 
ferasen enthält, welche die Bildung phenolischer Steroidglucuronide katalysieren. Der Nachweis von Glucuronyltransferasen gelang sowohl in der Mikrosomen-Fraktion als auch in der Cytoplasma-Fraktion (105000 g-Überstand). Ähnliche Befunde waren bereits früher mit Zellfraktionen des Dünndarms vom Menschen erhoben worden (6). Während das lösliche Enzym der Magenschleimhaut ausschließlich die $17 \beta$-ständigen, alkoholischen Hydroxylgruppen der phenolischen Steroide (17 $\beta$-Östradiol und Östriol) glucuronidierte, reagierte das mikrosomale Enzym nicht nur mit der $17 \beta$-ständigen, sondern auch mit der $16 \alpha$-ständigen Hydroxylgruppe von Östriol. Weder mit $17 \beta$-Östradiol noch mit Östriol als Substrat erfolgte eine Glucuronidierung der phenolischen Hydroxylgruppe am C-Atom 3; auch Östron ergab nach Inkubation mit den Enzympräparationen kein Östron-3-monoglucuronid. Aus diesen Ergebnissen kann auf eine Gruppenspezifität der mikrosomalen und cytoplasmatischen Glucuronyltransferasen der menschlichen Magenschleimhaut geschlossen werden. In diesem Punkte unterscheiden sich die Glucuronyltransferasen des Magens von denjenigen des Dünndarms (6): Die intestinalen Enzyme glucuronidieren sowohl die phenolische Hydroxylgruppe als auch die alkoholischen $\mathrm{Hy}$ droxylgruppen von Östrogenen $(5,6)$. Ob das Fehlen einer Glucuronidierung der phenolischen Hydroxylgruppe auf die Magenschleimhaut beschränkt ist, bedarf weiterer Untersuchungen; obgleich SLAuJNWHITE, LICHTMAN und SANDBERG (11) in Leberhomogenaten des Menschen ausschließlich eine $16 \alpha$-Glucuronidierung von Östriol fanden, konnten wir kürzlich sowohl in der Mikrosomen- als auch in der Cytoplasma-Fraktion der
Leber des Menschen eine Glucoronidierung der phenolischen Hydroxylgruppe von Östron nachweisen (12). Bei Verwendung von $17 \beta$-Östradiol als Substrat ist die Bildungstate des $17 \beta$-Monoglucuronids - bezogen auf die eingesetzte Eiweißmenge - in der MikrosomenFraktion etwa $25 \mathrm{mal}$ größer als in der CytoplasmaFraktion. Wesentlich geringere Unterschiede in der Bildung des $17 \beta$-Monoglucuronids durch das mikrosomale und cytoplasmatische Enzym wurden bei Verwendung von Östriol beobachtet; darüber hinaus glucuronidiert das mikrosomale Enzym die 16 $\alpha$-Hydroxygruppe in deutlich größerem Umfange als die $17 \beta$ Hydroxygruppe.

Über die physiologische Bedeutung der Glucuronidierung von Östrogen in der Magenschleimhaut läßt sich noch nichts Endgültiges aussagen. Man kann annehmen, $\mathrm{da} B$ die in der Magenschleimhaut gebildeten Östrogenglucuronide über die abführenden Magenvenen und die Pfortader direkt in die Leber gelangen. Damit werden die im Magen resorbierten und glucuronidierten Östrogene ebenfalls dem enterohepatischen Kreislauf der Östrogenglucuronide, über den früher bereits berichtet worden ist (13), zugeführt. Die Tatsache, daß der Magen bei der Resorption von Steroiden eine Rolle spielt, dürfte im Zusammenhang mit der oralen Verabreichung von Östrogenen von Interesse sein.

Die vorliegende Untersuchung wurde mit Unterstützung der Deutschen Forschungsgemeinschaft durchgeführt. Der eine von uns (W. H.) dankt der Deutschen Forschungsgemeinschaft für ein Stipendium. Herrn Dr. K. DAHM sind wir für wertvolle Hinweise und Fräulein Christa SChröder für ihre Mitarbeit zu Dank verpflichtet.

\section{Literatur}

1. Smith, E. R. und H. Breuer, Biochem. J. 88,168 (1963). 2. Breuer, H. und D. Wessendorf, Hoppe-Seyler's $Z$. physiol. Chem. 345, 1 (1966). - 3. Dahm, K., H. Breuer und M. Lindlau, Hoppe-Seyler's Z. physiol. Chem. 345, 139 (1966). - 4. DAHM, K. und H. Breuer, Biochim. biophysica Acta (Amsterdam) 113, 404 (1966). - 5. Dahm, K. und H. Breuer, Biochim. biophysica Acta (Amsterdam) 128, 306 (1966). - 6. DAHM, K. und H. Breuer, diese Z. 4, 153 (1966). - 7. Glucuronic Acid, free and combined. Hrsg. von Durton, G. J., Academic Press, New York and London,
1966. - 8. Stevenson, I. H. und G. J. Dutron, Biochem. J. 82, 330 (1962). - 9. Folin, O. und V. Crocalteu, J. biol. Chemistry 73, 627 (1927). - 10. DAHM, K. und H. BreurR, Acta endocr. (K'hvn.) 52, 43 (1966). - 11. SLAUNWhite, W. R. JR., M. A. LichtMAN und A. A. SANDBERG, J. Clin. Endoct. (Springfield) 24, 638 (1964). - 12. RAO, G. S. und H. BREUER, unveröffentlichte Versuche. - 13. Breuer, H. und K. Dahm, 12. Symp. d. Dtsch. Ges. f. Endokr., Springer, Heidelberg 1967, S. 280.

Prof. Dr. rer. nat. H. Breuer Abt. f. Klin. Chem. und Biochemie der Chirurg. Univ.-Klinik und Poliklinik 53 Bonn, Venusberg 